\title{
Scalability Performance for Low Power Wide Area Network Technology using Multiple Gateways
}

\author{
N.A. Abdul Latiff ${ }^{1}$, I.S. Ismail ${ }^{2}$ \\ Faculty of Ocean Engineering Technology and Informatics \\ University Malaysia Terengganu \\ Kuala Terengganu, Malaysia
}

\author{
M. H. Yusoff ${ }^{3}$ \\ Faculty of Informatics and Computing \\ Universiti Sultan Zainal Abidin \\ Kuala Terengganu, Malaysia
}

\begin{abstract}
Low Power Wide Area Network is one of the leading technologies for the Internet of Things. The capability to scale is one of the advantage criteria for a technology to compare to each other. The technology uses a star network topology for communication between the end-node and gateway. The star network topology enables the network to support a large number of end-nodes and with multiple of gateways deployed in the network, it can increase the number of end nodes even more. This paper aims to investigate the performance of the Low Power Wide Area Network Technology, focusing on the capability of the network to scale using multiple gateways as receivers. We model the network system based on the communication behaviours between the end-node and gateways. We also included the communication limit range for the data signal from the end-node to successfully be received by the gateways. The performance of the scalability for the Low Power Wide Area Network Technology is shown by the successfully received packet data at the gateways. The simulation to study the scalability was done based on several parameters, such as the number of end-nodes, gateways, channels and also application time. The results show that the amount of successfully received data signal at gateway increased as the gateways, application time and channel used increased.
\end{abstract}

Keywords-Low power wide area network; scalability; simulation; multiple gateways

\section{INTRODUCTION}

For the past few years, the advances in the development of Internet-of-Things (IoT) speed up the growth for new, innovative, convenience, and economic benefit applications. The things are any devices holding the ability of sensing, computing, and exchanging information with other devices by communicating via the internet [1]. The technology inspired by IoT is believed to be able to enhance the effectiveness of energy consumption, resources management, productivity, and environmental monitoring. The IoT has spread its importance to various domains which previously has a limitation in multiple possible applications, for example, real-time environmental, remote health-care, industrial control, productions systems, smart city, and transportation [2].

Developers have worked on many applications for various uses in the IoT, and specific applications require specialized technology to work accordingly. Traditional short-range communication technologies such as Bluetooth and ZigBee are not ideal for applications that require long-range communication. While cellular technology can provide broader coverage than conventional radio technology, it requires much energy to operate, which is not ideal for low power applications.

Recent advancement in applications that require smaller size device, low power consumption, and cost-effective have shown positive progress in the development of a new communication technology known as the Low Power Wide Area Network (LPWAN). The new technology becomes a complement to conventional communication technology such as cellular and short-range wireless technologies by augmenting a better functionality and requirement for IoT applications. The unique features of LPWAN technology such as high coverage, low bandwidth, and low power consumption, are in line with the requirements of IoT applications that only need to transmit small data sizes remotely.

LPWAN technology is designed to support billions of devices for the various applications of IoT. The technology uses a star topology architecture in which multiple end-nodes communicate directly to the gateway [3]. However, numerous end-nodes transmit the data signal to the gateway and this cause traffic overload, and eventually, there will be data signal loss at the gateway. Increasing the number of gateways can reduce the data signal overload by the single gateway. Even so, the scalability analysis frequently only used a single gateway to study the performance of the LPWAN.

In this study, the performance of the LPWAN in the capability to scale using multiple gateways. The development and simulation of the proposed network model were done based on the collision behaviour of the data signal from the end-node at the gateway using the MATLAB platform. The organization of this paper is as follow: In Section 2, related work on the previous study was presented. Then, the introduction of Low Power Wide Area Networks was presented in Section 3. Next, the proposed network model was discussed in section 4. In Section 5, the simulation procedure and parameters were discussed, and result and discussion were presented in Section 6. Finally, conclude the paper in conclusion.

\section{RELATED WORK}

The previous work on several studies on model development for LPWAN has been conducted previously for a better understanding of LPWAN's ability to scale. This section presents the previous studies on several works focusing on modelling and scalability of LPWAN. 
Georgiou and Raza [3] present the study of modelling of LPWAN to analyse the capability of the technology to scale. The authors used stochastic geometry framework to model the performance of the Long-Range (LoRa) network by using a single gateway. The study proposed an outage probability model which occurs at the gateway called outage condition.

Example of studies following the outage probability model by the authors in [3] was presented in [4]-[6]. The authors in [4] used time diversity to increase the probability of successful packet delivery from the sensor nodes to the gateways. While in [5], the authors presented the paper based on the model in [3]. It included the effect of co-spreading factor (co-SF) interference and inter-SF interference as the model in [3] did not consider the interferences factor in the model. The model presented in [6] is the extending of the outage models from [3] for diversity techniques.

The study of the scalability of LPWAN was presented in [7] by M. C. Bor, U. Roedig, T. Voigt, and J. M. Alonso. This paper investigates the number of transmitters that LoRa network can support. The authors developed LoRa simulator called LoRaSim, which is used to study the scalability of LoRa network. Following the model and simulator from [7], several studies focus on the performance of LPWAN in terms of scalability [8]-[10]. The authors in [8] used LoRa communication model based on [7] to develop further an improved version of LoRaSim called EXPLoRa. Meanwhile, the authors in[10] used the model and simulator from [7] to study the performances of LoRaSim on three different simulation parameters; SF, bandwidth, and coding rate.

The authors in [9] also developed a LoRa model similar to the model from [7]. The scalability of the LoRa network was studied by observing the most significant possible number of LoRa transmitter while satisfying the average packet success probability. The other model was developed by the authors in [11] to study the scalability of LoRa technology. The model used LoRa interference behaviour for the development of the data signal collision model. Meanwhile, the authors in [12] develop LoRaWAN simulator study the scalability of the LPWAN. The development of the packet collision model was inspired by the collision model from [11] to determine the behaviours of the data signal collision and the capture effect. The investigation in [13] shows improvement in the network scalability when using a method which assigning the SF used by the end-nodes in the network.

\section{LOW POWER WIDE AREA NETWORK}

Low Power Wide Area Network is a wireless communication technology that enables end nodes to communicate over long distance using low bit rates and low energy consumption [14][15][16]. Previous studies have shown that LPWAN technology enables the final node to communicate with gates over a distance of 3 kilometres for urban areas, while more than 10 kilometres for rural areas [17]. Additionally, in the line of sight circumstance, the last node data signal can reach a gateway located 20 kilometres away [18] can still reach the gateway as far as 30 kilometres, as reported in [19].
The ability of end-nodes to communicate remotely with a gateway is based on two main special features of the LPWAN, the star network topology and modulation technique. The LPWAN device mostly operates in the unlicensed Industrial, Scientific and Medical (ISM) bands at 169, 433, 868/915 $\mathrm{MHz}$, and $2.4 \mathrm{GHz}$ [20]. However, these frequency values [21][22] depend on the region in which the technology is being used.

Dynamic progress in LPWAN technology development has created many LPWAN-based applications and solutions in the market. The current most known LPWAN technologies are Sigfox and Semtech. The Sigfox technology uses three main components for the communication, which are Ultra Narrow Band radio technology, Binary Phase Shift Keying and Gaussian Frequency Shift Keying modulation. Typically, depending on the region, the ISM band used by the technology is at 868 to $869 \mathrm{MHz}$ and 902 to $928 \mathrm{MHz}$. Sigfox devices are capable of sending small data with 12 bytes of maximum data size for uplink data while 8 bytes of downlink using the Lightweight protocol. Altogether, the Sigfox frame uses 26 bytes, with 12 bytes of load data and 14 bytes for protocol overhead. This protocol overhead is smaller than conventional LPWAN technology, which applies more significant size protocol overheads to transmit data. [23].

In addition to Sigfox, Semtech also developed the LPWAN technology known as LoRa Technology. The technology is designed for a combination of remote, low power consumption, and secure small-size data transmission. It also operates on an unlicensed SUB-GHz ISM band using a so-called chirp spread spectrum (CSS) modulation to optimize power consumption and broader communications networks. LoRa Technology uses the combination of two layers; the physical layer is known as LoRa for the connectivity and the MAC layer known as LoRaWan.

\section{NETWORK MODEL}

This section describes the proposed network model to study the scalability of LPWAN. The communication model in this study mimics the communication protocol between end-node and gateway for scalability study purposes. The following are assumptions for behaviour of data signal from end-node to be received by the gateway based on [3], [7], [11].

\section{A. The Interference Conditions}

In this model, the end-nodes are group into two types which known as reference node and interference node. The reference node is current end-node transmitting data to the gateway at present. While, the interference node refers to others end-nodes beside the reference node that transmitting data signal before, present, or after the reference node transmitting data signal. The received status of data signal for the reference and interruption nodes at gateway can determine whether data signal is successfully received based on the collision condition.

Data signal interruption between the interference and reference nodes are assumed to base on three main parameters; SF, channel and transmission time. If data signals arrive at the gateway from the reference and interference nodes which use the same SF and channel, then all data signal 
are considered unsuccessfully received by the gateway. The gateway will receive all data signals if the SF and channel used are different. Data signal in this condition is said to be orthogonal to each other. Table I provides detail of the interference condition for both reference and interference nodes.

Data signal interference happens when both of the reference and interference nodes have the same SF and channel. However, data signal can be successfully received by the gateway if both data signal of the reference and interference nodes are being downloaded by the gateway, which passes the preamble time of data signal. Fig. 1 illustrates all possible interference conditions by the endnodes.

Data signal for the interference node in Case 1 and 6 are successfully received by the gateway as there is no data signal collision with the reference node. In Case 2, data signal from the reference node has successfully received to the gateway. Data signal arrives at the time where the preamble of data signal for the interference node already being downloaded by the gateway. In this situation, both of data signals from the reference and interference node are successfully downloaded by the gateway. This situation is also fit for Case 6 , where the roles of the reference and interference nodes exchange. When data signal from the reference node arrives during the preamble of data signal from interference node is being downloaded, both of data signals are assumed to be not received by the gateway as shown in Case 3. This condition is the same as Case 4, and Case 5, where the roles of reference and interference node exchange. Table II shows the received status of the interference and reference node at the gateway.

\section{B. SF Selection}

In this study, the SF selections used in the network model for the end-nodes was inspired by [2]. The selection of the SF depends on the distance between the end-node and the gateway. When gateway received data signal from the endnode, it also records the RSSI and SNR value of data signal. Typically, the RSSI and SNR values increase when the distance between the end-node and gateway increases. However, the data signal may attenuate depending on the condition of line-of-sight between the end-node and gateway, which results in increasing the recorded RSSI and SNR values. The end-node requires a higher SF to transmit data signal to the gateway, depending on data signal condition [11].

From previous study, the assumption made for the endnode which located far away from the gateway will use the SF of 12. Data signal is expected to be able to reach the gateway. However, data signal cannot be received by a gateway if the location of the end-node is located too far due to data signal attenuation. It is reasonable to have a limit distance between end-node and gateway for data signal to successfully receive by the gateway. Table III shows the selection of SF value for the data transmission base on the distance between the endnode and gateway. The assumption for the distance between the end-node and gateway is 2 kilometres for each SF. When the distance is over 12 kilometres, the signal is lost and did not received by the gateway.
TABLE. I. INTERFERENCE CONDITIONS OF THE DATA SIGNAL

\begin{tabular}{|l|l|l|}
\hline SF & Channel & Condition \\
\hline Same value & Same value & Interference \\
\hline Same value & Different value & No Interference \\
\hline Different value & Same value & No Interference \\
\hline Different value & Different value & No Interference \\
\hline
\end{tabular}

\begin{tabular}{|c|c|c|c|c|c|c|c|}
\hline Case 1 & $\mathrm{Ni}$ Preamble & Payloc & & & & & \\
\hline Case 2 & $\mathrm{Ni}$ & Preamble & Pay & load & & & \\
\hline Case 3 & & $\mathrm{Ni}$ & Prean & \begin{tabular}{l|l} 
nble & Payl
\end{tabular} & & & \\
\hline \multirow[t]{2}{*}{\begin{tabular}{|l|} 
Case 4 \\
\end{tabular}} & & & $\mathrm{Ni}\lceil$ & Preamble & Payload & & \\
\hline & & & $\mathrm{Nr}$ & Preamble & Payload & & \\
\hline Case 5 & & & & $\mathrm{Ni}$ Pream & \begin{tabular}{|l|l|} 
le & Payload \\
\end{tabular} & & \\
\hline Case 6 & & & & & $\mathrm{Ni} \stackrel{\text { Preamble }}{ }$ & Payload & \\
\hline \multirow[t]{2}{*}{$\begin{array}{l}\text { Case } 7 \\
\end{array}$} & & & & & $\mathrm{Ni}$ & \begin{tabular}{|l|} 
Preamble \\
\end{tabular} & Payload \\
\hline & & & & Time & & & \\
\hline
\end{tabular}

Fig. 1. Diagram of Reference and Interference Node based on Time.

TABLE. II. STATUS OF REFERENCE NODE AND INTERFERENCE NODE

\begin{tabular}{|l|l|l|}
\hline Case & Status Ni & Status Nr \\
\hline 1 & Receive & Receive \\
\hline 2 & Receive & Receive \\
\hline 3 & Loss & Loss \\
\hline 4 & Loss & Loss \\
\hline 5 & Loss & Loss \\
\hline 6 & Receive & Receive \\
\hline $\mathbf{7}$ & Receive & Receive \\
\hline
\end{tabular}

TABLE. III. SF SELECTION OF THE END-NODE

\begin{tabular}{|l|l|l|}
\hline $\mathbf{S F}$ & RSSI $\mathbf{( d B m})$ & Distance $(\mathbf{k m})$ \\
\hline $\mathbf{7}$ & $(-124,-100)$ & $<2$ \\
\hline $\mathbf{8}$ & $(-129,-124)$ & $2-4$ \\
\hline $\mathbf{9}$ & $(-130,-129)$ & $4-6$ \\
\hline $\mathbf{1 0}$ & $(-133,-130)$ & $6-8$ \\
\hline $\mathbf{1 1}$ & $(-135,-133)$ & $8-10$ \\
\hline $\mathbf{1 2}$ & $(-137,-137)$ & $10-12$ \\
\hline $\mathbf{1 2}$ & $(-140,-137)$ & $12-14$ \\
\hline$\sim$ & $\sim$ & $>14$ \\
\hline
\end{tabular}

\section{Gateways Location}

In this study, multiple gateways were used to receive the data signal from the end-nodes. The number of gateways used are 2, 4, and 6. The model used 2-dimensional network field with the same length, $(L \times L)$. The locations of the gateway were based on the length $(L)$ of the network field. Let say the coordinate of a gateway, $G W(g)=(G W x, G W y)$ where $g$ is the number of the gateway, $G W x$ is the coordinate in x-axis and $G W y$ is the coordinate in y-axis. Then the locations of the gateway were based on the number of getaways used and were given in below equations.

If gateways used are 2

$$
\begin{aligned}
& G W(1)=(L / 4, L / 2) \\
& G W(2)=(L-L / 4, L / 2)
\end{aligned}
$$


If gateways used are 4

$$
\begin{aligned}
& G W(1)=(L / 4, L / 4) \\
& G W(2)=(L-L / 4, L / 4) \\
& G W(3)=(L / 4, L-L / 4) \\
& G W(4)=(L-L / 4, L-L / 4)
\end{aligned}
$$

If gateways used are 6

$$
\begin{aligned}
& G W(1)=(L / 4, L / 4) \\
& G W(2)=(L / 2, L / 4) \\
& G W(3)=(L-L / 4, L / 4) \\
& G W(4)=(L / 4, L-L / 4) \\
& G W(5)=(L / 2, L-L / 4) \\
& G W(6)=(L-L / 4, L-L / 4)
\end{aligned}
$$

\section{Simulation}

The performance of proposed model is executed via simulation using MATLAB platform. Let say there are $N$ numbers of end-nodes distributed randomly in $L x \times L y$ twodimensional network field. The end-nodes are assumed to use specific SF based on the distance between the end-node and the gateway $d$, as discussed in the previous section. Let $D(n)=$ $(x(n), y(n))$ be the coordinate of the distributed end-nodes and $G W(g)=(G W x, G W y)$ be the coordinate of the gateway location. Where $n=\{1,2,3 \ldots, N)$ and $\mathrm{g}$ is the number of gateways. Then, the distance for the end-node $j$ from the gateway $g$ is defined as;

$\mathrm{d}(\mathrm{GW}(\mathrm{g}), \mathrm{D}(\mathrm{n}))=$

$\left[(G W x(\mathrm{~g})-\mathrm{x}(\mathrm{n}))^{2}+(G W y(\mathrm{~g})-\mathrm{y}(\mathrm{n}))^{2}\right]^{1 / 2}$

Typically, in LPWAN, one end-node can transmit data signal and is received by multiple gateways. The network will decide which optimal gateway for the next data transmission of the end-node based on the link strength at the gateway [11]. The received signal strength at the gateway is mainly related to the distance between the node and the gateway. The proposed model used these conditions for the end-node to choose the nearest gateway to transmit the data signal. Then, the network will assign the SF based on the distance for the end-node between the end-node and the gateway.

Additionally, the gateway also randomly assigned the channel $(\mathrm{CH}(\mathrm{n}))$ for the end-nodes in the range of [1, $\mathrm{CH}]$, where $\mathrm{CH}$ is the total number of channels. The starting time is assigned randomly for the end-nodes to start transmitting the packet data to mimic the real application of the end-nodes. Starting time (ST(n)) is randomly chosen by the end-nodes based on the range time [0, Application time]. Application time is time for end-node to transmit next data signal after complete transmitting the current data signal.

$$
\begin{aligned}
& \mathrm{CH}(\mathrm{n})=\operatorname{rand}([1, \mathrm{CH}]) \\
& \mathrm{ST}(\mathrm{n})=\operatorname{rand}([0, \text { Application time }]
\end{aligned}
$$

Then, the end-node starts to transmit the packet data to the corresponding gateway based on its starting time. When the end-node complete transmitting the packet data, the end-node will set the new starting time (New_ST(n)) with the combination of the starting time, time-on-air (ToA), and processing time (PT). Processing time is time for the end-node to process the data for the next transmission sequel in the range of $[0,1.000 \mathrm{~s}]$. Note that, the maximum time for endnodes to process the data is assumed to be $1 \mathrm{~s}$. ToA is the time for data signal from the end-node to successfully receive by the gateway. However, it depends on the size of the payload, bandwidth, SF and code rate used by the end-node for data transmission. Refer to [24] for more information on ToA.

$\mathrm{PT}(\mathrm{n})=[0,1.000 \mathrm{~s}]$

New_ST $(n)=S T(n)+T o A(n)+P T(n)$

At each of the gateway, data signal is successfully received based on the interference conditions as discussed in the previous section. The simulation is run based on round. Each round ends if all the end-node complete transmitting data signal to the gateway. Once the run has reached the designated total run, the simulation stops. Then, the program calculates the percentage of received packet data (PPD). PPD is the percentage of the total received data signal at gateway over the total number of data signal transmission from end-node. Table IV shows the parameter used in the simulation.

The excerpt of the program used in the MATLAB are given below:

Randomly place $\mathrm{N}$ end-nodes in network field

Randomly set CH, ST and PT

Calculated the distance for each end-node

Set the SF for the end-nodes

Set the end-nodes to the corresponding GW based on the distance

for round $=1: 50$

for $\mathrm{Nr}=1: \mathrm{N}$

for $\mathrm{Ni}=1: \mathrm{N}$

if $\mathrm{CH}(\mathrm{Nr})==\mathrm{CH}(\mathrm{Ni}) \& \& \mathrm{SF}(\mathrm{Nr})==\mathrm{SF}(\mathrm{Ni})$

Both data signals not received by $\mathrm{GW}$

Status received $=0$

else if Case 1, Case 2, Case 6 or Case 7

Both data signal received by $\mathrm{GW}$

Status received $=1$

else if Case 3, Case 4 or Case 5

Both data signals not received by $\mathrm{GW}$

Status received $=0$

end

end

end

Set New_ST for the end-node

end

end

$\mathrm{PPD}($ round $)=\operatorname{sum}($ Status received $) / \mathrm{N} * 100$

end

$\mathrm{PPD}=\operatorname{sum}(\mathrm{PPD}) /$ round 
TABLE. IV. PARAMETERS FOR THE SIMULATION

\begin{tabular}{|l|l|}
\hline Parameters & Values \\
\hline Size of network field & $24000 \mathrm{~m} \times 24000 \mathrm{~m}, 48000 \mathrm{~m} \times 48000 \mathrm{~m}$ \\
\hline Number of end-nodes, $\mathrm{N}$ & $\begin{array}{l}100,200,300,400,500,600,700,800, \\
900,1000,1500,2000\end{array}$ \\
\hline Number of gateways & $2,4,6$ \\
\hline Packet payload size & 50 bytes \\
\hline Frequency & $868 \mathrm{MHz}$ \\
\hline Bandwidth & $125 \mathrm{kHz}$ \\
\hline Coding rate & $4 / 5$ \\
\hline SF & $7,8,9,10,11,12$ \\
\hline Channel & 1,8 \\
\hline Application time $(\mathrm{s})$ & 600,3600 \\
\hline Total Round & 50 \\
\hline
\end{tabular}

\section{RESULTS AND ANALYSIS}

Fig. 2 below shows the example of 500 end-nodes (represented by blank round shapes) with four gateways (represented black round shapes) in the $24000 \mathrm{~m}$ x $24000 \mathrm{~m}$ network field. The different colour of the blank round shapes indicates the end-nodes that transmitted data signal to the respective gateway.

Next, Fig. 3 to 5 shows the percentage of the end-node per gateway. The percentage value is calculated based on the average total number of the end-node transmitted to the gateway for 50 rounds. The average number of end-node per gateway varied as the total number of the gateway increased. The end-nodes are located randomly in the network field while the locations of the gateways are fixed. The unbalance numbers of end-node per gateway will affect data signal throughput of the gateway. For example, as shown in Fig. 5, the total number of end-nodes transmitting data signal to gateway 1, 3, 4, and 6 are higher compared to gateway 2 and 5 . The higher number of end-node per gateways will have a higher chance for data signal of end-nodes to collide with each other during data transmission.

In the simulation results, the effect of different parameters can be observed on the PPD using the proposed model. Fig. 6 to 9 show the results for PPD using one and 8-Channels with different application time and size of the network field. Overall, the PPD value decreases when the number of endnodes increases. Observations of this reduction in PPD occur because the amount of data signal from the sensor node rises, resulting in more data signal arriving at the gateways. These increase the chance of data signal to collide with each other's resulting in data signal loss at the gateway.

The overall value of PPD shown in Fig. 6 increases when the number of gateways increases. Increasing the number of gateways will decrease the throughput load by a single gateway. Besides, increasing the number of channel in the network will also increasing the PPD value. Referring to the interference conditions of data signal, data signal with the different channel will avoid the collision.

Meanwhile, the result in Fig. 7 shows a similar pattern as in Fig. 6. Increasing value of application time results in increasing the PPD value. This is shown in Fig. 6 with the application time of 600s, while in Fig. 7 with the application time of 3600s. In a single channel with two gateways, the PPD value of the application time of 3600s gives a similar result to the PPD value when using 8-channel with two gateways with an application time of 300s. The higher application time increases the time difference of the starting time between the end-nodes (refer to equation 15). This increment in time minimizes the number of end-nodes that has the same or similar starting time. Then, the chance for data signal to collide with each other is also reduced.

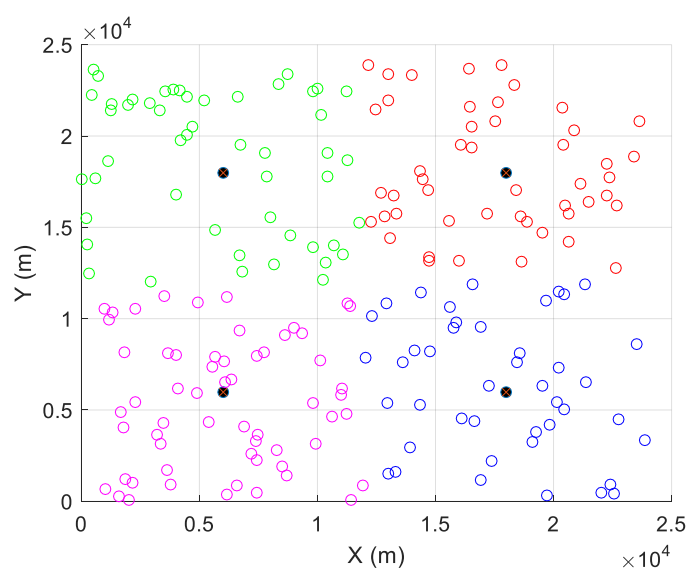

Fig. 2. Location of the End-Nodes and the Gateways.

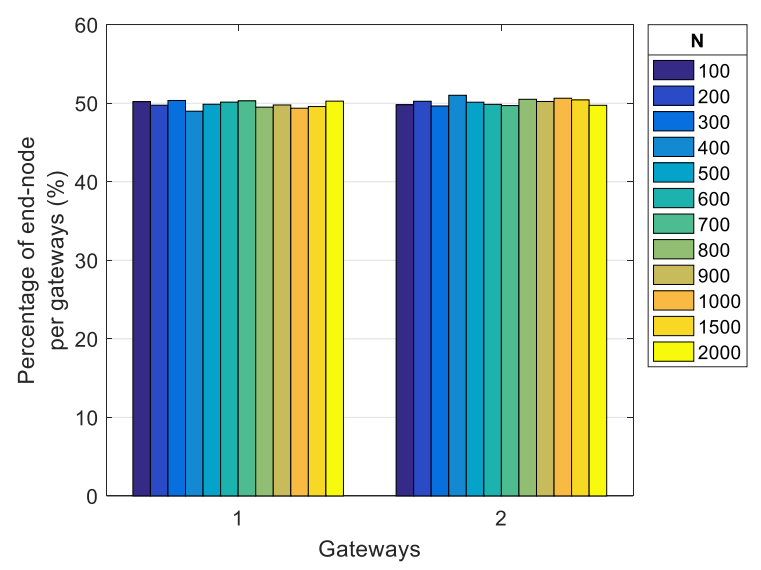

Fig. 3. Percentage of the End-Node Per Gateways using 2 Gateways.

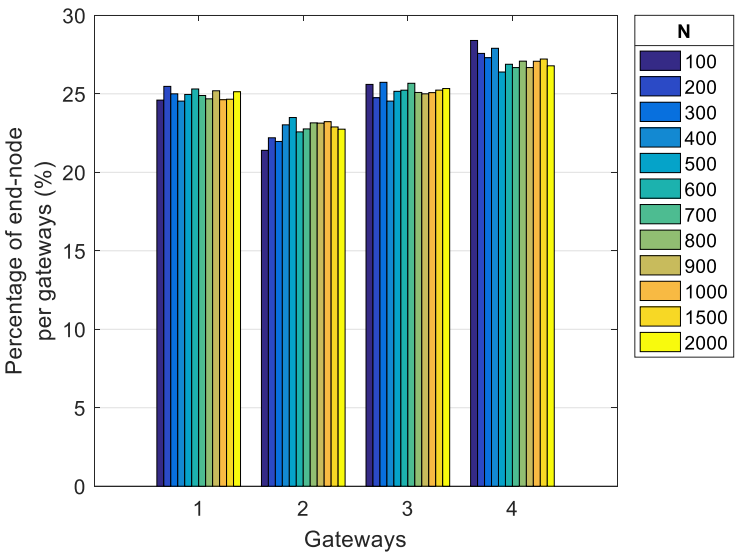

Fig. 4. Percentage of the End-Node Per Gateways using 4 Gateways. 


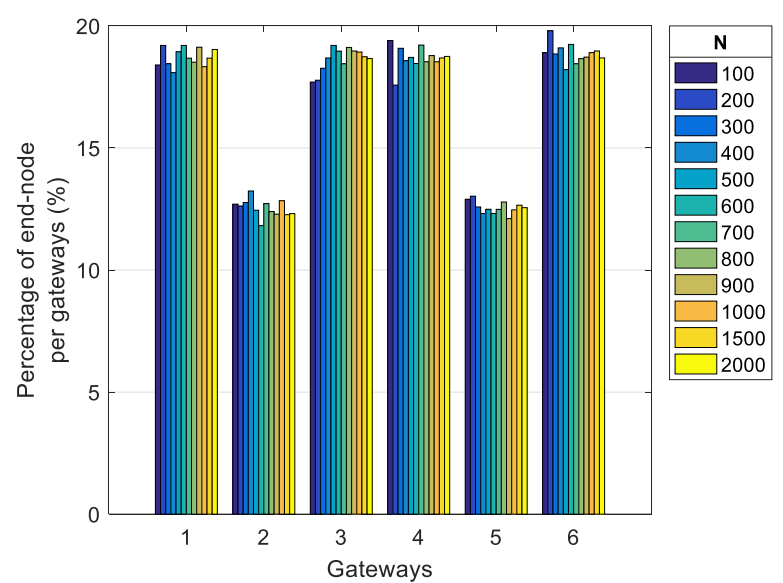

Fig. 5. Percentage of the End-Node Per Gateways using 6 Gateways.

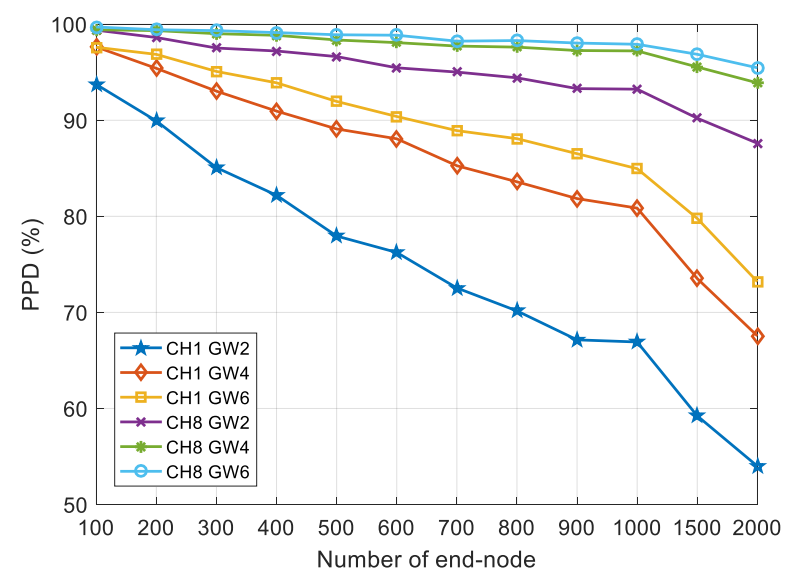

Fig. 6. PPD using Application Time of 600s in 24000mx24000m Network Field.

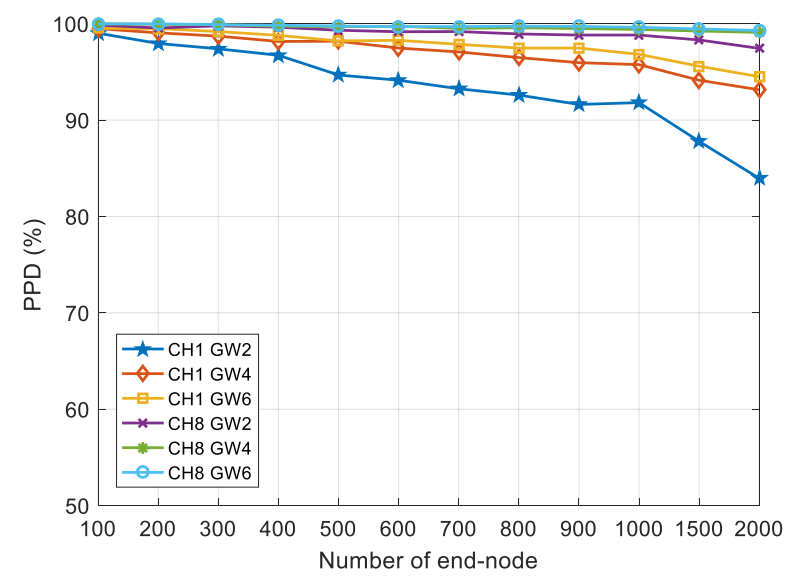

Fig. 7. PPD using Application Time of 3600s in 24000mx24000m Network Field.

Although the PPD value increases when either the number of gateway or channel increases, increasing the channel gives better performance compared to increasing the number of gateways. Meanwhile, increasing the amount of application time gives better results of PPD compared to increasing the number of gateway or channel.

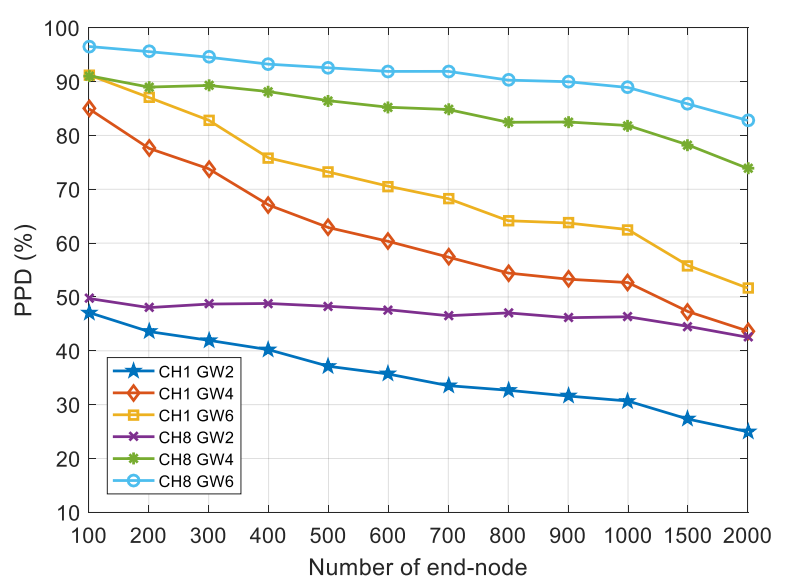

Fig. 8. PPD using Application Time of $600 \mathrm{~s}$ in $48000 \mathrm{mx} 48000 \mathrm{~m}$ Network Field.

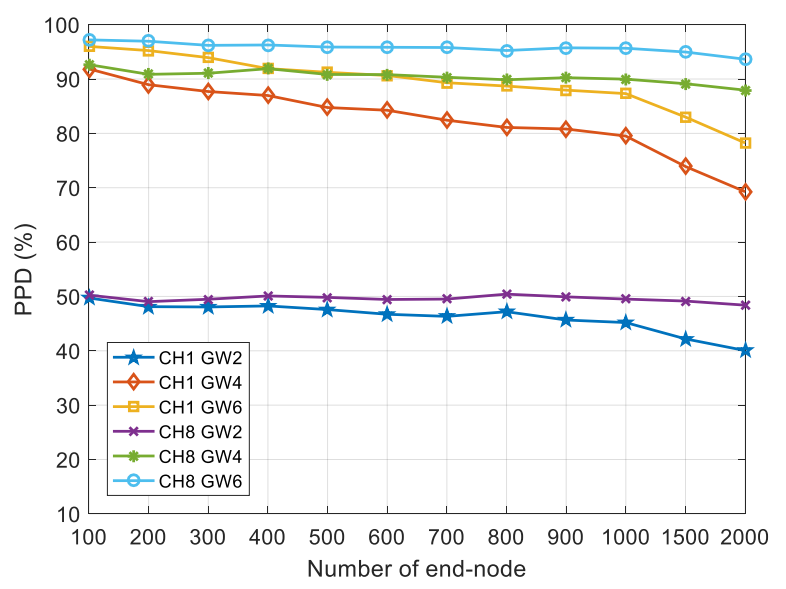

Fig. 9. PPD using Application Time of 3600s in 48000mx48000m Network Field.

Fig. 8, and 9 show the results use the similar parameter as in Fig. 6 and 7 but in double size of the network field. Overall, the PPD value also decreases as the number of end-nodes increases which are similar to the previous results but with a lower PPD value. Increasing the amount of channel or the value application time results in increasing the PPD value. The PPD value when using only two gateways is at $50 \%$ and below. This effect is due to increasing the size of the network field. This decrement value of PPD indicates that only half of data signal from the end-node received by the gateways. The gateway solely collected data signal when the location of the one-nodes was in the range of the set limit distance. However, the value of PPD increases when using more gateways to receive data signal.

\section{CONCLUSION}

In this paper, the development and simulation of a comprehensive model of LPWAN to study the scalability using MATLAB simulator is presented. This model includes several assumptions based on the behaviour of LPWAN communication between the end-nodes and gateways, such as the interference conditions of data signal and the selection of the Spreading Factor and the application time. The results show that increasing number of end-nodes, decreases the 
value of PPD. However, the PPD value increases when the number of gateways, channel and application time increase. The locations of the gateways are directly influencing total number of the end-nodes. The placements of these gateways affect the total number of the end-nodes per gateway. Data signal collisions are more likely to occur when more endnodes transmitting to a single gateway. Deploying more gateways may overcome this problem. However, in real-time application, increasing the number of gateways will double the cost. Meanwhile, selection of high performance of LPWAN devices is important in order to support high number of channels.

The future scope of the current proposed work can be developed by choosing the optimal locations of the gateways. This optimal location of the gateways should be able to increase the data delivery to the gateways compared to this current proposed location of the gateways when using a similar network environment.

\section{ACKNOWLEDGMENT}

The authors would like to be obliged to University Malaysia Terengganu and Ministry of Education Malaysia for providing financial assistance under project Fundamental Research Grant Scheme (FRGS) no. 59466.

\section{REFERENCES}

[1] K. C. Serdaroglu and S. Baydere, "WiSEGATE: Wireless Sensor Network Gateway framework for internet of things," Wirel. Networks, vol. 22, no. 5, pp. 1475-1491, Jul. 2016.

[2] I. S. Ismail, N. A. A. Latiff, and N. A. A. Aziemah, "Performance Analysis of Data Recovery via Application Layer for LPWAN," in 2019 IEEE 89th Vehicular Technology Conference (VTC2019-Spring), 2019, vol. 2019-April, pp. 1-5.

[3] O. Georgiou and U. Raza, "Low Power Wide Area Network Analysis: Can LoRa Scale?," IEEE Wirel. Commun. Lett., vol. 6, no. 2, pp. 162165, Apr. 2017.

[4] A. Hoeller, R. D. Souza, O. L. A. Ĺopez, H. Alves, M. De Noronha Neto, and G. Brante, "Exploiting Time Diversity of LoRa Networks Through Optimum Message Replication," Proc. Int. Symp. Wirel. Commun. Syst., vol. 2018-Augus, pp. 1-5, 2018.

[5] A. Mahmood, E. Sisinni, L. Guntupalli, R. Rondon, S. A. Hassan, and M. Gidlund, "Scalability Analysis of a LoRa Network Under Imperfect Orthogonality," IEEE Trans. Ind. Informatics, vol. 15, no. 3, pp. 14251436, Mar. 2019.

[6] A. Hoeller, R. D. Souza, O. L. Alcaraz Lopez, H. Alves, M. de Noronha Neto, and G. Brante, "Analysis and Performance Optimization of LoRa Networks With Time and Antenna Diversity," IEEE Access, vol. 6, no. c, pp. 32820-32829, 2018.

[7] M. C. Bor, U. Roedig, T. Voigt, and J. M. Alonso, "Do LoRa LowPower Wide-Area Networks Scale?," Proc. 19th ACM Int. Conf. Model. Anal. Simul. Wirel. Mob. Syst. - MSWiM '16, no. November, pp. 5967, 2016.

[8] F. Cuomo, M. Campo, A. Caponi, G. Bianchi, G. Rossini, and P. Pisani, "EXPLoRa: Extending the performance of LoRa by suitable spreading factor allocations," Int. Conf. Wirel. Mob. Comput. Netw. Commun., vol. 2017-Octob, 2017.

[9] D. Zorbas, G. Z. Papadopoulos, P. Maille, N. Montavont, and C. Douligeris, "Improving LoRa Network Capacity Using Multiple Spreading Factor Configurations," 2018 25th Int. Conf. Telecommun. ICT 2018, pp. 516-520, 2018.

[10] A. Lavric and V. Popa, "Performance Evaluation of LoRaWAN Communication Scalability in Large-Scale Wireless Sensor Networks," Wirel. Commun. Mob. Comput., vol. 2018, pp. 1-9, Jun. 2018.

[11] J. Haxhibeqiri, F. Van den Abeele, I. Moerman, and J. Hoebeke, "LoRa Scalability: A Simulation Model Based on Interference Measurements," Sensors, vol. 17, no. 6, p. 1193, May 2017.

[12] A. M. Yousuf, E. M. Rochester, B. Ousat, and M. Ghaderi, "Throughput, Coverage and Scalability of LoRa LPWAN for Internet of Things," 2018 IEEE/ACM 26th Int. Symp. Qual. Serv. IWQoS 2018, 2019.

[13] A. Tiurlikova, N. Stepanov, and K. Mikhaylov, "Method of Assigning Spreading Factor to Improve the Scalability of the LoRaWan Wide Area Network," Int. Congr. Ultra Mod. Telecommun. Control Syst. Work., vol. 2018-Novem, pp. 1-4, 2019.

[14] D. H. Kim, J. Y. Lim, and J. D. Kim, "Low-Power, Long-Range, HighData Transmission Using Wi-Fi and LoRa," in 2016 6th International Conference on IT Convergence and Security (ICITCS), 2016, pp. 1-3.

[15] P. Neumann, J. Montavont, and T. Noel, "Indoor deployment of lowpower wide area networks (LPWAN): A LoRaWAN case study," in 2016 IEEE 12th International Conference on Wireless and Mobile Computing, Networking and Communications (WiMob), 2016, pp. 1-8.

[16] D. Kim and M. Jung, "Data Transmission and Network Architecture in Long Range Low Power Sensor Networks for IoT," Wirel. Pers. Commun., vol. 93, no. 1, pp. 119-129, Mar. 2017.

[17] M. Centenaro, L. Vangelista, A. Zanella, and M. Zorzi, "Long-range communications in unlicensed bands: the rising stars in the IoT and smart city scenarios," IEEE Wirel. Commun., vol. 23, no. 5, pp. 60-67, Oct. 2016.

[18] C. Pham, "Low-cost, low-power and long-range image sensor for visual surveillance," in Proceedings of the 2nd Workshop on Experiences in the Design and Implementation of Smart Objects - SmartObjects '16, 2016, pp. 35-40.

[19] J. Petajajarvi, K. Mikhaylov, A. Roivainen, T. Hanninen, and M. Pettissalo, "On the coverage of LPWANs: range evaluation and channel attenuation model for LoRa technology," in 2015 14th International Conference on ITS Telecommunications (ITST), 2015, pp. 55-59.

[20] I. I.S., A. L. N.A., R. F.Z., and A. A. S., A Review on Performances Evaluation of Low Power Wide Area Networks Technology. Springer Singapore, 2019.

[21] A.-I. Pop, U. Raza, P. Kulkarni, and M. Sooriyabandara, "Does Bidirectional Traffic Do More Harm Than Good in LoRaWAN Based LPWA Networks?," no. April, Apr. 2017.

[22] I. LoRa Alliance, "LoRaWANTM 1.0.2 Regional Parameters," San Ramon, CA, 2017.

[23] Sigfox, "Radio Technology Keypoints." [Online]. Available: https://www.sigfox.com/en/sigfox-iot-radio-technology. [Accessed: 09Jan-2018].

[24] Semtech, "SX1272/3/6/7/8: LoRa Modem Design Guide," 2013. [Online]. Available: https://www.semtech.com/uploads/documents/ LoraDesignGuide_STD.pdf. [Accessed: 09-Apr-2018]. 